\title{
Effect of Different Bracket Prescriptions on Orthodontic Treatment Outcomes Measured by Three-dimensional Scanning
}

\author{
Tomohiro Soboku, Etsuko Motegi and Kenji Sueishi
}

Department of Orthodontics, Tokyo Dental College, 2-9-18 Kanda-Misakicho, Chiyoda-ku, Tokyo 101-0061, Japan

Received 11 May, 2018/Accepted for Publication 4 July, 2018

Published Online in J-STAGE 10 April, 2019

\begin{abstract}
Various types of bracket are currently available, and different prescriptions are provided for the same type of tooth. There are no definite criteria, however, on which to base bracket selection. The purpose of this study was to investigate differences in the angulation and inclination of orthodontically aligned teeth when using different prescription brackets. Thirty patients undergoing orthodontic treatment for maxillary protrusion and crowding were enrolled. After orthodontic treatment with a pre-adjusted appliance, angulation and inclination were measured on dental casts obtained from these patients (10 each with 0.022 MBT, 0.022 Roth, or 0.018 Roth brackets). The dental casts were scanned and digitized using a 3-dimensional (3-D) scanner and measured with 3-D model measurement software. A significant difference was observed in the mean angulation of the mandibular canine between the $0.022 \mathrm{MBT}\left(5.81^{\circ}\right)$ and 0.018 Roth groups $\left(9.07^{\circ}\right)$. Greater mesial inclination was observed in the 0.018 Roth group. No significant difference was observed in any of the other regions measured. Differences in bracket prescription showed no clinical influence on treatment outcomes. A significant difference was observed in the mandibular canine $(\mathrm{p}<0.05)$, suggesting that consideration is required when selecting brackets.
\end{abstract}

Key words: $\quad$ Pre-adjusted appliance — Bracket prescription - 3-D scanning Post-treatment angulation and inclination

\section{Introduction}

The aim of orthodontic treatment is to achieve the best possible balance among its various goals, which include obtaining a functional occlusal relationship, esthetically pleasing dentition and facial features, and longterm occlusal stability. One criterion for

This paper was a thesis submitted by Tomohiro Soboku to the Graduate School of Tokyo Dental College. 


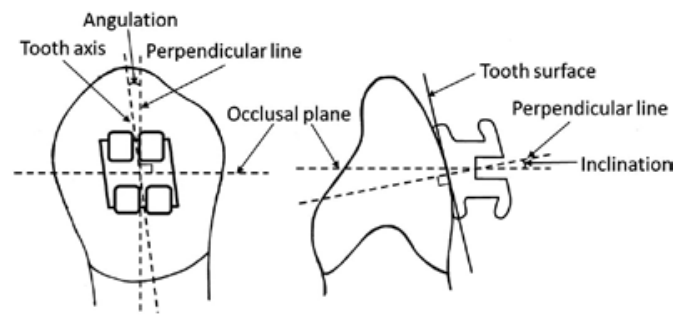

Fig. 1 Characteristics of pre-adjusted appliances

Pre-adjusted appliances have in-built prescriptions of angulation and inclination which are optimized for average cases.

achieving this is whether the ideal axial inclination of the teeth has been secured or not after completion of treatment. This has led to the development of pre-adjusted appliances that allow 3-dimensional (3-D) control of the positioning of the teeth, which offers more consistent and effective treatment results (Fig. 1) ${ }^{11)}$.

Historically, Andrews appears to have been the first to present the Straight Wire Appliance (SWA), in 1972. He was the first to propose the concept of 'Six Keys to Normal Occlusion', which was derived from common features observed in dental casts obtained from 120 individuals with normal occlusion who had not undergone any orthodontic treatment ${ }^{1}$. The angulation, inclination, and in/out values for each of the teeth in the models were measured. The extent of bracket slot grinding and direction were then adjusted using the results as target values. This concept was then subsequently commercialized. The concept developed by Andrews is characterized by the creation of various types of bracket which are based not only on anatomical values, but also take into account slot shape (i.e. torque in base), degree of malocclusion, and even degree of wire bending required as a result of treatment method, such as tooth extraction or non-extraction. Orthodontists were instructed to use different types of bracket depending on each patient's needs.

The high degree of personalization which characterized treatment by this method made clinicians feel that bracket selection was diffi- cult to understand and too complicated, however. Therefore, in 1987, an attempt was made to simply this method with the development of several criteria that could be applied to most cases (consistent angulation and inclination volumes), which resulted in the Roth prescription bracket ${ }^{14)}$. Influenced by gnathology, Roth proposed functional occlusion based on the concept that orthodontic care required functional checks. This supported the Roth prescription and led to its acceptance by many orthodontists.

When using the Roth Appliance for the canines, the Andrews prescription was used if tooth extraction was required. This was done as greater care had to be taken to avoid overcorrection in such cases than would otherwise have been necessary with other prescriptions. This was done to suppress inclination and rotation toward the tooth extraction site, and significant angulation would be prescribed.

After considering outcomes with use of the Andrews SWA bracket in a large number of patients in the 1990s, McLaughlin et al. developed the MBT appliance, which is based on sliding mechanics and a weak sustainable orthodontic force ${ }^{9}$. The MBT bracket was designed to induce greater labial inclination in the maxillary anterior teeth and increase lingual inclination in the mandibular anterior teeth. These prescriptions suppressed lingual inclination in the maxillary anterior teeth during their anterior retraction and labial inclination in the mandibular anterior teeth when applying a curve of Spee to the wire. The MBT bracket was set as a constant volume, as with the Roth bracket. In terms of slot size, the $0.022 \times 0.028$-slot bracket was used by various pioneers in orthodontics such as Angle and Tweed, although some orthodontists currently use $0.018 \times 0.025$-slot brackets to apply weak orthodontic force with narrow arch wires. Other orthodontists use $0.022 \times 0.028$-slot brackets for other reasons, such as wire stiffness, to make the sliding mechanics smoother ${ }^{2)}$.

In 2002, Keim et al. presented a study on slot size and bracket prescription as chosen by orthodontists in the United States. They 
Table 1 Prescription for angulation and inclination

\begin{tabular}{|c|c|c|c|c|c|c|}
\hline \multirow[b]{2}{*}{ type of tooth } & \multicolumn{3}{|c|}{ angulation $\left({ }^{\circ}\right)$} & \multicolumn{3}{|c|}{ inclination $\left({ }^{\circ}\right)$} \\
\hline & MBT & Roth & difference & MBT & Roth & difference \\
\hline \multicolumn{7}{|l|}{ Maxillary } \\
\hline 1 & 4 & 5 & 1 & 17 & 12 & 5 \\
\hline 2 & 8 & 9 & 1 & 10 & 8 & 2 \\
\hline 3 & 8 & 13 & 5 & 0 & -2 & 2 \\
\hline 5 & 0 & 0 & 0 & -7 & -7 & 0 \\
\hline 6 & 0 & 0 & 0 & -14 & -14 & 0 \\
\hline 7 & 0 & 0 & 0 & -14 & -14 & 0 \\
\hline \multicolumn{7}{|l|}{ Mandibular } \\
\hline 1 & 0 & 2 & 2 & -6 & -1 & 5 \\
\hline 2 & 0 & 2 & 2 & -6 & -1 & 5 \\
\hline 3 & 3 & 7 & 4 & 0 & -11 & 11 \\
\hline 5 & 2 & -1 & 3 & -17 & -22 & 5 \\
\hline 6 & 0 & -1 & 1 & -20 & -17 & 3 \\
\hline 7 & 0 & -1 & 1 & -10 & -30 & 20 \\
\hline
\end{tabular}

reported that $54.2 \%$ of those surveyed used $0.022 \times 0.028$-slots (0.022-slots), $40.5 \%$ used $0.018 \times 0.025$-slots (0.018-slots), $4.3 \%$ used other slot sizes, and $4.3 \%$ used slots of 2 different dimensions $(0.018 \times 0.025$-slots for the anterior teeth and $0.022 \times 0.028$-slots for the molars). ${ }^{8)}$

In 2003, Sheridan reported that prescription of pre-adjusted brackets was chosen on the basis of the specific technique to be used, and that approximately $47 \%$ of those surveyed were in favor of prescribing Roth brackets. In addition, the majority of those surveyed used $0.022 \times 0.028$-slots with $0.019 \times 0.025$-stainless steel wire as the finishing wire. ${ }^{16)}$

At present, various angulation and inclination prescriptions are available, and although the prescription can differ for the same type of tooth, no clear criteria have been suggested on which to base bracket selection, including bracket slot size. Therefore, our goal was to investigate which prescriptions integrated into 3 types of bracket yielded consistent posttreatment results. These treatment outcomes were assessed in the canines, which exhibit great variation.

\section{Materials and Methods}

\section{Materials}

All patients enrolled in this study underwent orthodontic treatment for maxillary protrusion and crowding at the orthodontic departments of Tokyo Dental College Chiba Hospital or Suidobashi Hospital. A total of 30 patients ( 4 men and 26 women) with plaster dental casts meeting the conditions given below were selected for inclusion. The MBT (Victory, Clarity, 3M, St. Paul, Minn, USA) or Roth (Mini Twin, Sprit Twin, ORMCO, Orange, CA, USA) brackets were used. These are two of the most widely used bracket types worldwide. The age of the selected patients on completion of orthodontic treatment ranged from 13 years and 6 months to 34 years and 3 months (average, 18 years and 7 months).

1) Bracket type: 0.022 MBT 10 cases (20 teeth), 0.022 Roth 10 cases (20 teeth), 0.018 Roth 10 cases (20 teeth)

The bracket prescriptions used in orthodontic treatment are shown in Table 1.

2) Finishing wire size: 0.018 -slot: $0.016 \times 0.022$ 

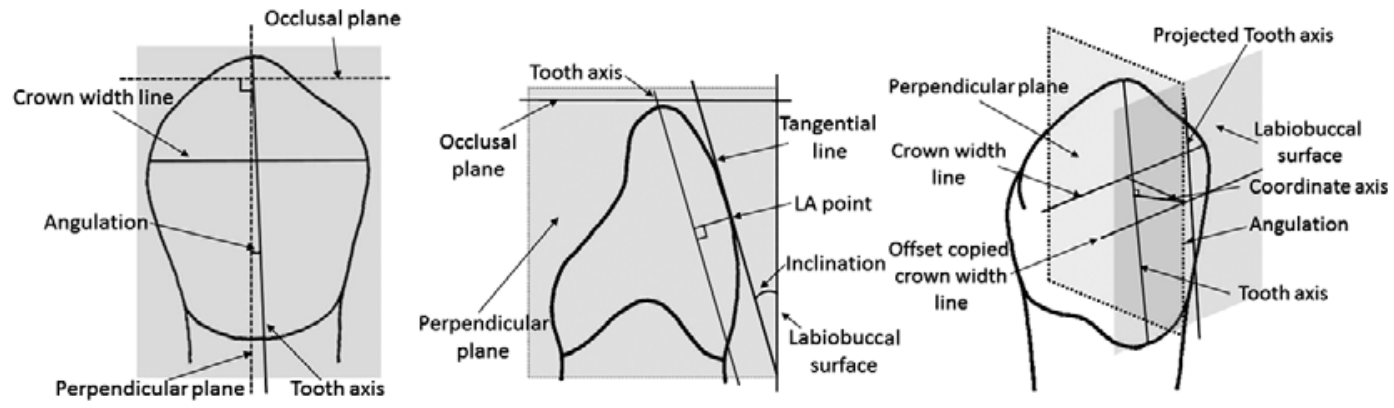

Fig. 2 Diagram of angulation and inclination measurement

To measure angulation, tooth axes were projected onto labiobuccal surface. Projected axis and perpendicular plane to occlusal plane. Tangential line was established on LA point; angle between this tangent and labiobuccal surface was marked as crown inclination.

inch, 0.022-slot: $0.019 \times 0.025$-inch

3) Maxillary and mandibular first premolar extraction

4) $1.0 \leq \mathrm{ANB} \leq 7.0$

5) Overjet: $>+1 \mathrm{~mm}$

6) Skeletal pattern: Mesiofacial

7) Molar relation: Class I or II

8) Restorative treatment restricted to pits and fissures and no prosthetic crowns covering the cusps

\section{Methods}

\section{1) Measurement method}

To input images of dental casts, a 3-D scanner (3Shape R700 Orthodontic Scanner, 3Shape, Denmark) was used to scan the shape of the tooth cast on completion of orthodontic treatment.

After trimming the digital cast with the included software (Ortho Viewer, 3Shape, Denmark), a 3-D dentition image of a parallel cast was created and exported as an STL data file. The 3-D dentition image obtained through this process was opened with Workstation's (Dell Precision T3400, USA) 3-D point cloud processing software (Imageware13, UGS Inc., USA). The 3-D image obtained was checked to ensure that there were no blind areas ${ }^{18)}$.

First, the occlusal plane was established with the maxillary and mandibular teeth in occlusion using the following the 3 reference points: an anterior reference point located at the center of the maxillary and mandibular central incisors where they overlap; and two more posterior reference points located bilaterally at the center of the maxillary and mandibular first molars where they overlap.

Angulation and inclination in the maxillary and mandibular central incisors, lateral incisors, canines, second premolars, first molars, and second molars was then measured on the basis of the 3-D data from the dental casts obtained on completion of orthodontic treatment in the 0.022 MBT, 0.022 Roth, and 0.018 Roth groups using the method described below. The mean values for each tooth were calculated based on two separate measurements.

(1) Angulation (mesiodistal axial inclination of the teeth) (Figs. 2,3)

Angulation was measured using the method described by Fuma $e t a l^{6)}$. First, the tooth axes were determined. The tooth axis for the anterior teeth was defined as the line connecting the center of the incisal edge and the center of the labiocervical region; the tooth axis of the premolars was defined as the line connecting the buccal cusp and the center of the buccocervical region, while the tooth axis for the molars was defined as the line connecting the buccocervical region and the midpoint between the mesiobuccal cusp and the distobuccal cusp. The crown width line was then 


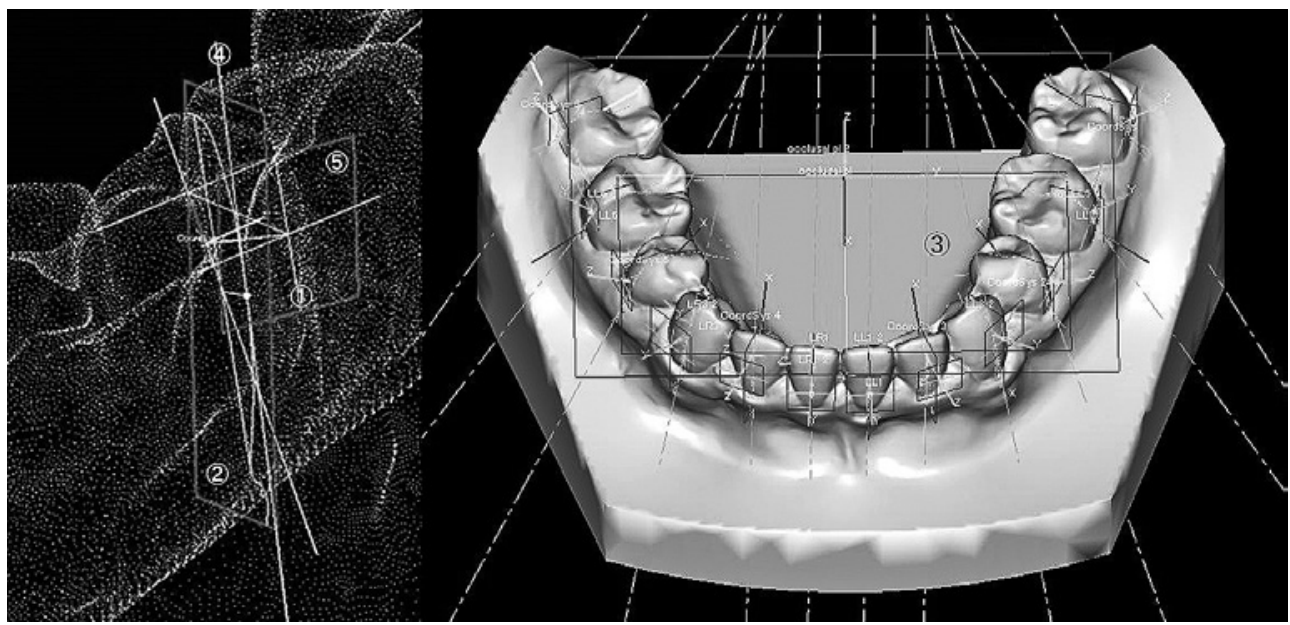

Fig. 3 Three-dimensional dental cast images

Angulation measurements were performed on 3-dimensional dental cast images. Angle of (1) and (2) formed crown angulation.

Inclination measurements were performed on 3-dimensional dental cast images. Angle of (4) and (5) formed crown inclination.

(1) Tooth axes projected onto labiobuccal surface, (2) Perpendicular plane to occlusal plane, (3) Occlusal plane, (4) Tangential line established on LA point, (5) Labiobuccal surface

established as the line connecting the mesiodistal contact points of each tooth. Next, a perpendicular line was drawn between the crown width line and an offset copied crown width line. A plane perpendicular to the occlusal plane was then created (perpendicular plane). Next, a plane was established orthogonal to the perpendicular plane, and the labiobuccal surface created. The tooth axes were subsequently projected onto this labiobuccal surface, with the angle of the projected axis and perpendicular plane forming the crown angulation.

(2) Inclination (buccolingual axial inclination of the teeth) (Figs. 2,3)

Inclination was determined based on the method of Fukagawa $e t a l^{5}$. First, the orientation of the sagittal plane of the tooth axis was established by creating a coordinate axis at the intersection of the aforementioned offset copied crown width line and the tooth axis perpendicular line. Next, a crown-shaped curvature was made on the established tooth axis and projected onto the perpendicular plane together with the tooth axis. A perpen- dicular line was then created on the crownshaped curve from the midpoint of the tooth axis, and the point where the crown-shaped curve and line intersect the labiobuccal crown midpoint (LA point) was determined. Finally, a tangential line was established on the LA point, and the angle between this tangent and the labial surface marked as the crown inclination.

The reproducibility of the measurements of angulation and inclination was checked. Angulation and inclination measurements were repeated 5 times on the maxillary central incisors, as representative of the anterior teeth, and the first molars, as representative of the molars to establish the standard deviation. The standard deviation was 0.081-0.769. The measurement error was sufficiently small, and reproducibility was deemed high.

2) Statistical analysis

The mean and standard deviation of the angulation and inclination of all the teeth, excluding the maxillary and mandibular first premolars, were calculated. A one-way analysis of variance (ANOVA) was used to compare 


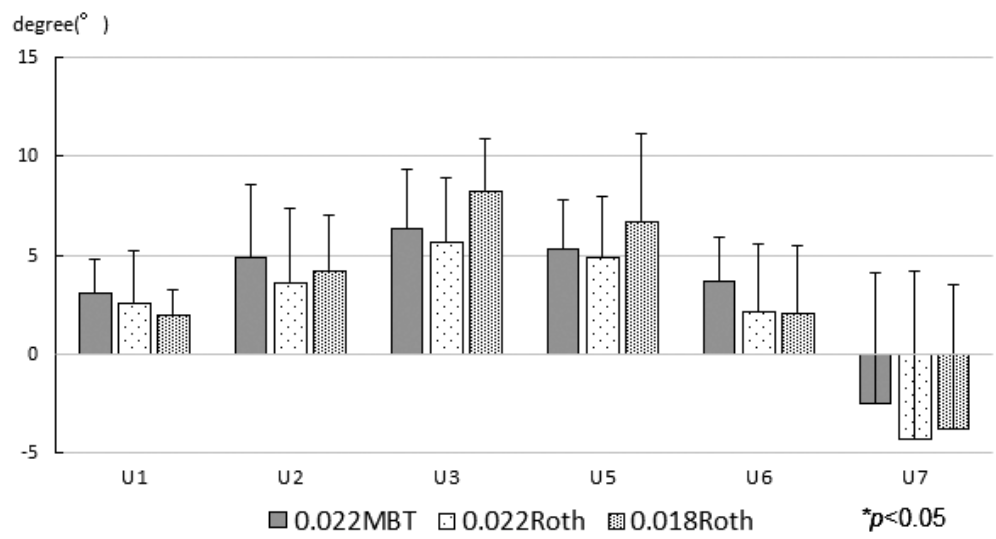

Fig. 4 Maxillary angulation measurement

Results of angulation measurement in maxilla revealed no significant differences among 3 groups.

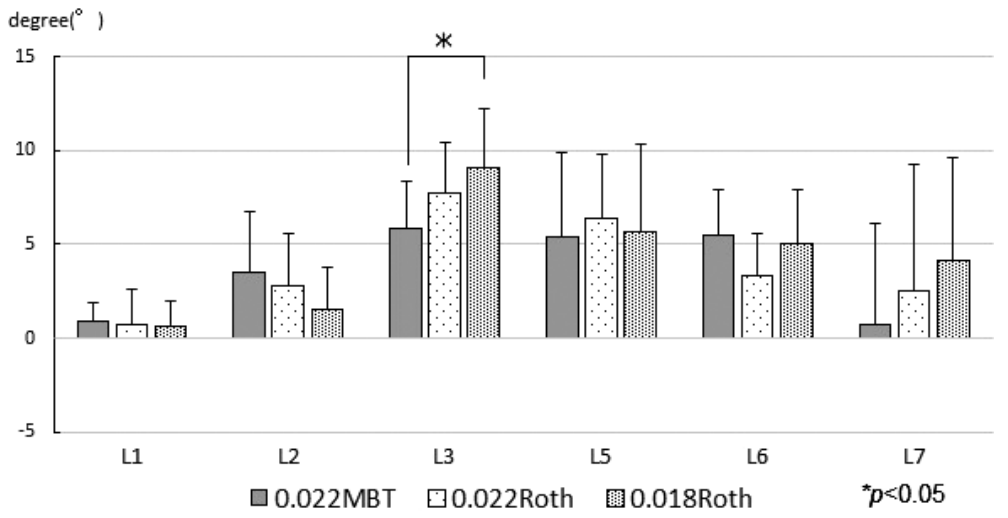

Fig. 5 Mandibular angulation measurement

Results of angulation measurement in mandible revealed significant differences between mean values for mandibular canines between 0.022 MBT and 0.018 Roth groups $\left(5.81^{\circ}\right.$ vs. $9.07^{\circ}$, respectively).

the 0.022 MBT, 0.022 Roth, and 0.018 Roth groups; multiple comparisons were made using the Bonferroni correction. The level of significance was set at $\mathrm{p}<0.05$.

The protocol of this study was approved by the Ethics Committee of Tokyo Dental College (approval number: 468).

\section{Results}

Figures 4-7 show the mean, standard deviation, and ANOVA results for the angulation and inclination of all the teeth, excluding the maxillary and mandibular first premolars.

1. Angulation (Figs. 4, 5; Table 2)

Differences in bracket prescription (Table 1) were large for the canines. Although there were no significant differences among the 3 
Table 2 Angulation measurement

\begin{tabular}{|c|c|c|c|c|c|c|}
\hline \multirow[b]{2}{*}{ type of tooth } & \multicolumn{2}{|c|}{$0.022 \mathrm{MBT}$} & \multicolumn{2}{|c|}{ 0.022Roth } & \multicolumn{2}{|c|}{ 0.018Roth } \\
\hline & Mean & $\mathrm{SD}$ & Mean & $\mathrm{SD}$ & Mean & $\mathrm{SD}$ \\
\hline \multicolumn{7}{|l|}{ Maxillary } \\
\hline 1 & 3.09 & 1.75 & 2.62 & 2.64 & 2.03 & 1.25 \\
\hline 2 & 4.87 & 3.69 & 3.66 & 3.69 & 4.27 & 2.73 \\
\hline 3 & 6.35 & 3.00 & 5.67 & 3.22 & 8.30 & 2.60 \\
\hline 5 & 5.34 & 2.48 & 4.88 & 3.07 & 6.75 & 4.39 \\
\hline 6 & 3.68 & 2.23 & 2.18 & 3.36 & 2.11 & 3.38 \\
\hline 7 & -2.48 & 6.63 & -4.22 & 8.45 & -3.75 & 7.28 \\
\hline \multicolumn{7}{|l|}{ Mandibular } \\
\hline 1 & 0.93 & 0.97 & 0.72 & 1.92 & 0.68 & 1.34 \\
\hline 2 & 3.52 & 3.24 & 2.83 & 2.72 & 1.53 & 2.24 \\
\hline 3 & 5.81 & 2.55 & 7.77 & 2.69 & 9.07 & 3.17 \\
\hline 5 & 5.37 & 4.54 & 6.43 & 3.42 & 5.68 & 4.65 \\
\hline 6 & 5.46 & 2.43 & 3.40 & 2.16 & 5.02 & 2.89 \\
\hline 7 & 0.71 & 5.39 & 2.51 & 6.73 & 4.18 & 5.47 \\
\hline
\end{tabular}

groups in the maxillary canines, a significant difference was observed between the mean values of the $0.022 \mathrm{MBT}$ and 0.018 Roth groups $\left(5.81^{\circ}\right.$ vs. $\left.9.07^{\circ}\right)$ in the mandibular canines. Greater mesial inclination was observed in the 0.018 Roth group. No significant difference was observed among the 3 groups in any other region.

Deviation in the maxillary and mandibular second molars was large, and distal inclination of the maxillary second molars with negative values was observed in all 3 groups.

\section{Inclination (Figs. 6, 7; Table 3)}

Differences in bracket prescription were the greatest for the mandibular canines (Table 1), although no significant difference was observed among the 3 groups. In addition, no significant difference was observed among the 3 groups in any of the regions measured. Strong lingual inclination of the mandibular molars was observed in the measurement results for bracket prescription in all 3 groups.

\section{Discussion}

\section{Research materials}

In investigating the effect of bracket prescription in detail, one important element of data collection is the elimination of confounding factors. Here, these factors included skeletal difference, occlusal force, ossein, root length, wire size (play), wire stiffness, bracket position, wire bending, elastics (mechanics), and ligation method.

Mittal et al. measured differences in the inclination of the anterior teeth in a Roth group and an MBT group with the bilateral maxillary first premolars extracted, an ANB angle of 1 to $5^{\circ}$, and a $0.019 \times 0.025$-inch stainless working arch wire in $0.022 \times 0.028$-inch bracket slots ${ }^{10)}$. The groups were limited to patients who did not meet the exclusion criteria of a non-extraction approach, extractions other than premolars, functional appliance treatment, headgear treatment, or orthognathic surgery. Their study cited materials used during treatment in addition to patient factors, and noted a loss of torque due to the method of ligation and/or differences in wire 


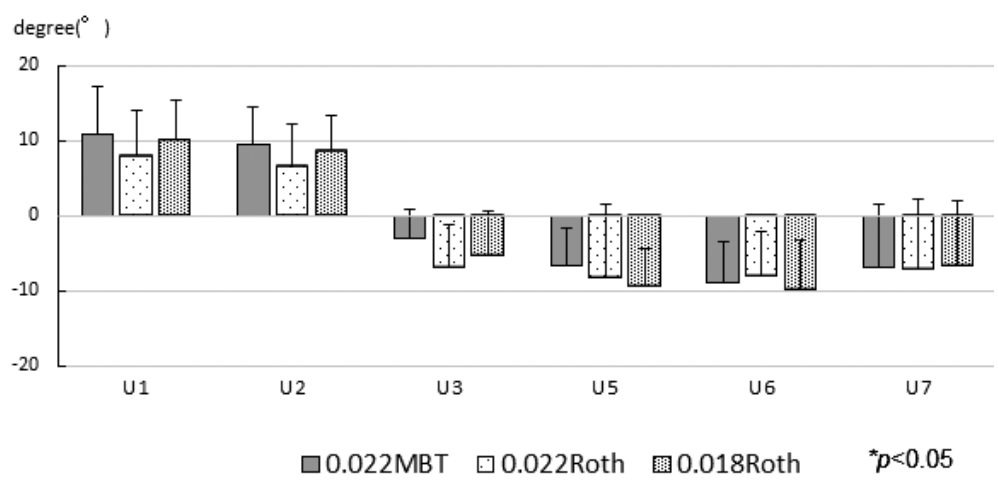

Fig. 6 Maxillary inclination measurement

Results of inclination measurement in maxilla revealed no significant differences among 3 groups.

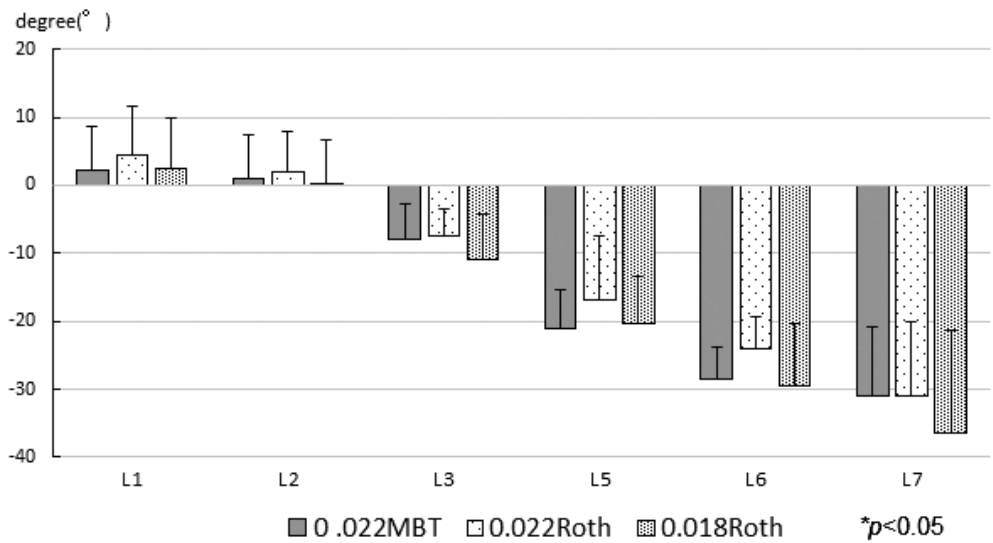

Fig. 7 Mandibular inclination measurement

Results of inclination measurement in mandible revealed no significant differences among 3 groups.

stiffness.

In the present study, horizontal and vertical skeletal effects, which are believed to arise from differences in occlusal force, were reduced by including patients with a mesiofacial pattern. Angle class III cases, where dental compensation due to skeletal discrepancy affects the anterior teeth during orthodontic treatment, which has a large effect on tooth inclination, were also excluded.

The ANB angle was also defined as $\geq 1^{\circ}$ to eliminate skeletal effects. In addition, extrac- tion cases that required more control of tooth inclination during anterior retraction were selected.

To compare the effect of bracket prescription among the 3 groups, wire size was restricted to a $0.016 \times 0.022$-inch finishing wire in a 0.018 -bracket slot or a $0.019 \times 0.025$ inch finishing wire in a 0.022 -bracket slot. The finishing wires were made of stainless steel or titanium molybdenum alloy. Moreover, types of bracket prescription other than those targeted here are also used at our hospital, and 
Table 3 Inclination measurement

\begin{tabular}{|c|c|c|c|c|c|c|}
\hline \multirow[b]{2}{*}{ type of tooth } & \multicolumn{2}{|c|}{$0.022 \mathrm{MBT}$} & \multicolumn{2}{|c|}{ 0.022Roth } & \multicolumn{2}{|c|}{0.018 Roth } \\
\hline & Mean & SD & Mean & SD & Mean & $\mathrm{SD}$ \\
\hline \multicolumn{7}{|l|}{ Maxillary } \\
\hline 1 & 11.00 & 6.21 & 8.09 & 5.89 & 10.20 & 5.22 \\
\hline 2 & 9.46 & 5.15 & 6.59 & 5.59 & 8.81 & 4.67 \\
\hline 3 & -2.97 & 3.93 & -6.85 & 5.61 & -5.11 & 5.76 \\
\hline 5 & -6.68 & 5.10 & -8.18 & 9.72 & -9.31 & 5.02 \\
\hline 6 & -8.99 & 5.52 & -7.84 & 5.82 & -9.78 & 6.45 \\
\hline 7 & -6.95 & 8.61 & -6.88 & 9.12 & -6.62 & 8.66 \\
\hline \multicolumn{7}{|l|}{ Mandibular } \\
\hline 1 & 2.27 & 6.46 & 4.47 & 7.11 & 2.57 & 7.23 \\
\hline 2 & 0.92 & 6.48 & 2.14 & 5.70 & 0.15 & 6.51 \\
\hline 3 & -7.99 & 5.25 & -7.40 & 3.93 & -10.80 & 6.57 \\
\hline 5 & -20.96 & 5.64 & -16.82 & 9.30 & -20.38 & 7.00 \\
\hline 6 & -28.62 & 4.80 & -23.93 & 4.49 & -29.48 & 9.10 \\
\hline 7 & -30.88 & 9.94 & -30.90 & 10.77 & -36.37 & 15.09 \\
\hline
\end{tabular}

not only typical cases with various facial patterns or prosthetic teeth, but also unusual cases with skeletal discrepancies or missing teeth are observed. Therefore, it was difficult to select preferable cases from the total of 13,581 patients initially considered.

\section{Three-dimensional model analysis}

The same 3-D model shape measuring apparatus as that used by Tanoi et $a l .{ }^{18)}$ and Fuma et al. was used here also ${ }^{6}$. Models have traditionally been measured using a direct manipulative method $^{15)}$; however, the recent application of computers and laser scanners in the field of dentistry has enabled more precise 3-D model shape measurement of the teeth.

Motohashi et al. reported that an x-axis of $0.07 \pm 0.07 \mathrm{~mm}$, y-axis of $0.07 \pm 0.06 \mathrm{~mm}$, and z-axis of $0.05 \pm 0.05 \mathrm{~mm}$ enabled accurate laser scanner measurement ${ }^{12)}$. The 3-D scanner used in the present study offers an accuracy of \pm 20 microns, which enables more accurate scanning than that which can be obtained with the non-contact, high-speed, 3-D shape-measuring apparatus (Non-Con- tact Shape Measuring System, VMS-100F, UNISN, Inc., Osaka, Japan) used by Tanoi $e t$ $a l^{18)}$, which offers accuracy of only \pm 50 microns.

In addition, to eliminate the possibility of human error during measurement, the teeth model images were enlarged on a computer and errors identified to improve accuracy.

\section{Results}

Pre-adjusted appliances were developed as a result of adjusting bracket design to consolidate prescriptions into a single type, in principle. Such consolidation into one type of prescription can be interpreted as a way of producing similar results without disparities in typical cases, and of yielding consistent outcomes. Moesi et al. conducted a questionnaire survey of 9 orthodontists to assess and compare the quality of results obtained from a study model of treatment outcomes in 20 patients receiving Roth prescriptions and 20 receiving MBT prescriptions ${ }^{11}$. Their assessment was based on the Incisor and Canine Aesthetic Torque and Tip scores. They found no statistically significant difference between 
the two bracket prescriptions, and thus concluded that bracket prescription had no effect on esthetic assessment.

The results of the present study showed no statistically significant difference in most of the comparisons. One possible reason for this was the play between the bracket slot and wire. According to Creekmore et al., a $0.016 \times 0.022$-inch wire offers $11.8^{\circ}$ of play with a 0.018 -slot bracket and a $0.019 \times 0.025$ inch wire offers $12.8^{\circ}$ of play with a 0.022 -slot bracket $^{3)}$. This suggests that the presence of play in inclination cancels out differences between groups. Thus, theoretically, bracket inclination prescriptions do not always exert all their effects depending on the amount of play between the slot and wire. When inclination is treated with a full-size arch wire, which is more likely to achieve full inclination in actual treatment, significant differences may be detected between the compared bracket prescriptions. However, in the present study, no materials incorporating a full-size wire were used as the aim was to compare appliances under more realistic slot and wire conditions, such as those that might be encountered in actual clinical practice. In fact, we believe that there should not be any statistically significant difference in typical cases.

The standard deviation in the results of the present study was also large. Germane $e t$ al. examined the effect of crown surface morphology on inclination and found that the former was not consistent in the same type of tooth between different patients ${ }^{7)}$. They reported that this variation in crown surface morphology gradually increased from the anterior teeth to the molars in both the maxilla and mandible. In addition, Van Loenen $e t$ al. reported that attaching brackets at from 2 to $4.5 \mathrm{~mm}$ from the incisal margin of the maxillary central incisors or canines might result in a mean $10^{\circ}$ of differential expression of torque on completion of treatment in the same patient when using one type of bracket system due to variation in crown morphology ${ }^{19)}$.

On the other hand, in the present study, a significant difference was observed in man- dibular canine angulation between the 0.022 MBT and 0.018 Roth groups. This was thought to be due to the effect of bracket prescription. There was a $4^{\circ}$ difference in the mandibular canine brackets that were compared in the present study, which was larger than that in the other regions. A $5^{\circ}$ difference in the maxillary canines was also observed, although the effect of the bracket was small and the difference not significant. Therefore, the usage of the 0.018 Roth is possibly effective in cases with canine distal tipping or a larger retraction space in the mandibular canines. A significant difference in mandibular canine angulation was also observed between the $0.022 \mathrm{MBT}$ and 0.018 Roth groups, in which different slot sizes were used; however, no significant difference was observed between the 0.022 Roth and 0.018 Roth groups. Detterline $e t$ al. assessed non-extraction patients using the American Board of Orthodontics Objective Grading System, and their results suggested that 0.018-slot brackets have a greater effect on angulation than 0.022-slot brackets. However, the effect of different slot sizes was small in the measurement results of the present study ${ }^{4)}$.

Furthermore, as the present study targeted maxillary and mandibular first premolar extraction cases, the patients required canine and anterior tooth traction. Therefore, in contrast to with anterior tooth and anteroposterior traction, the canines required buccolingual and anteroposterior traction to be implemented in an arch-shape in accordance with the dentition. The canines exhibit a different movement pattern than the other teeth, and this might have been the cause of the significant difference observed here. However, these significant differences in canine angulation but none in the maxillary canines might have been due to the larger roots of the maxillary canines. A large tooth root area can be disadvantageous in applying traction. Depending on the extent of crowding, a short traction distance and short period of treatment in the maxillary canines, which are larger than the lower canines, may make them resistant to bracket effects. 
Moreover, despite $0^{\circ}$ prescriptions in the maxillary second molars in all 3 groups in the present study, distal inclination was only observed in the maxillary second molars. This was probably due to a lack of rear eruption space for the second molars or an exaggerated curve of Spee associated with this as a result of choosing patients with crowding and maxillary protrusion. Compared with the other teeth, the standard deviation was also larger in the maxillary second molars in all 3 groups. This was probably because the second molar was the rearmost molar as an outcome of treatment and was therefore not easily controlled by the arch wire under cantilever conditions. The curve of Spee applied to the wire during retraction of the anterior teeth may have also had an effect in terms of the treatment mechanics. Individuals with normal occlusion have a gentle curve of Spee and exhibit distal inclination of the maxillary second molars with respect to the occlusal plane. These conditions may have reduced the effect of the appliance and caused distal inclination.

Furthermore, one earlier study described how the mandibular molars were shifted more lingually due to enhanced clenching strength associated with occlusal force ${ }^{17)}$. In other words, the effects of the bracket prescription in all 3 groups in the present study were smaller due to the strong combined effects of bracket-wire play and occlusal force, which caused greater lingual inclination than that of bracket prescription itself ${ }^{13,17)}$.

The conditions in the present study were set with a clinical perspective in mind. The results revealed no significant difference among the 3 types of bracket that were compared, underscoring that each of these brackets yields similar results and outcomes. Brackets are simply tools used to induce normal occlusion, and it is the patient's physical function that ultimately creates occlusion. Preadjusted appliances are highly useful devices for inducing this physical function. This suggests that it is important to have an understanding of the overall situation of treatment and the effects of bracket prescription when treating patients.

\section{Conclusions}

Angulation and inclination in dental casts obtained on completion of orthodontic treatment with different bracket prescriptions were measured. The results revealed that differences in bracket prescription did not clinically influence treatment outcomes. A significant difference was observed in mandibular canine angulation, however, between the $0.022 \mathrm{MBT}$ and 0.018 Roth groups, which suggests that consideration is required when selecting brackets.

\section{References}

1) Andrews LF (1972) The six keys to normal occlusion. Am J Orthod 62:296-309.

2) Cash AC, Good SA, Curtis RV, McDonald F (2004) An evaluation of slot size in orthodontic brackets-are standards as expected? Angle Orthod 74:450-453.

3) Creekmore TD, Kunik RL (1993) Straight wire: the next generation. Am J Orthod Dentofacial Orthop 104:8-20.

4) Detterline DA, Isikbay SC, Brizendine EJ, Kula KS (2010) Clinical outcomes of 0.018-inch and 0.022-inch bracket slot using the $\mathrm{ABO}$ objective grading system. Angle Orthod 80: 528-532.

5) Fukagawa H, Motegi E, Fuma A, Nomura M, Kano M, Sueishi K, Okano S (2010)Tooth inclination in elderly with many remaining teeth observed by 3-D imaging. Bull Tokyo Dent Coll 51:69-76.

6) Fuma A, Motegi E, Fukagawa H, Nomura M, Kano M, Sueishi K, Okano S (2010) Mesiodistal tooth angulation in elderly with many remaining teeth observed by 3-D imaging. Bull Tokyo Dent Coll 51:57-64.

7) Germane N, Bentley BE Jr, Isaacson RJ (1989) Three biologic variables modifying faciolingual tooth angulation by straightwire appliances. Am J Orthod Dentofacial Orthop 96: 312-319.

8) Keim RG, Gottlieb EL, Nelson AH, Vogel DS (2002) JCO study of orthodontic diagnosis and treatment procedures: part 1 results and trends. J Clin Orthod 36:553-568. 
9) McLaughlin RP, Bennett JC, Trevisi HJ (2002) Chapter 2, Systemized orthodontic treatment mechanics, Koga M, Himuro T, pp.13-14, Mosby, St Louis.

10) Mittal M, Thiruvenkatachari B, Sandler PJ, Benson PE (2015) A three-dimensional comparison of torque achieved with a preadjusted edgewise appliance using a Roth or MBT prescription. Angle Orthod 85:292-297.

11) Moesi B, Dyer F, Benson PE (2013) Roth versus MBT: does bracket prescription have an effect on the subjective outcome of preadjusted edgewise treatment? Eur J Orthod 35:236-243.

12) Motohashi N, Nunota A, Kuroda T (1997) Study on CAM system for dental model. J Stomatol Soc 64:427-435.

13) Okada D, Miura H, Hasegawa S (1998) The investigation of upper and lower posterior teeth in function-the three dimensional tooth displacement-. J Jpn Prosthod Soc 42: 279-286. (in Japanese)

14) Roth RH (1981) Functional occlusion for the orthodontist. J Clin Orthod 15:32-51,100-123, 174-198, 246-265.

15) Sebata E (1980) An orthodontic study of teeth and dental arch form on the Japanese normal occlusions. J Tokyo Dent Coll Soc 80:945-969. (in Japanese)

16) Sheridan IJ (2003) Reader's corner. J Clin Orthod 37:27-29.

17) Suzuki M, Miura H (2006) The relationship between three-dimensional occlusal force and tooth displacement depending on clenching force in function. JJpn Stomatological Soc 73: 79-89. (in Japanese)

18) Tanoi A, Motegi E, Sueishi K (2012) Change in dentition over 20 years from third decade of life. Orthod Waves 71:90-98.

19) Van Loenen M, Degrieck J, De Pauw G, Dermaut L (2005) Anterior tooth morphology and its effect on torque. Eur J Orthod 27: 258-262.

\section{Correspondence:}

Dr. Kenji Sueishi

Department of Orthodontics, Tokyo Dental College, 2-9-18 Kanda-Misakicho, Chiyoda-ku, Tokyo 101-0061, Japan

E-mail: sueishi@tdc.ac.jp 